\title{
Autoimmune lymphoproliferative syndrome with recurrent viral infections
}

INSERM

\section{Source}

INSERM. (1999). Orphanet: an online rare disease and orphan drug data base.

Autoimmune lymphoproliferative syndrome with recurrent viral infections.

ORPHA:275517

Autoimmune lymphoproliferative syndrome (ALPS) with recurrent viral infections is a rare genetic disorder characterized by lymphadenopathy and/or splenomeg aly and recurrent infections due to herpes viruses. 